\title{
sciendo
}

\section{Perspectives of the development of world economy in the blockchain conditions and Big Data}

\author{
Constantin ANGHELACHE \\ The Bucharest University of Economic Studies / "Artifex" University of Bucharest, Romania \\ actincon@yahoo.com \\ Mădălina-Gabriela ANGHEL \\ "Artifex" University of Bucharest, Romania \\ madalinagabriela anghel@yahoo.com \\ Gabriel Ștefan DUMBRAVĂ \\ The Bucharest University of Economic Studies, Romania \\ stefan.dumbrava@gmail.com \\ Daniel DUMITRU \\ The Bucharest University of Economic Studies, Romania \\ dumitru.teticdaniel@gmail.com
}

\begin{abstract}
Blockchain is a concept that tends to revolutionize the world of finance in a technological leap that allows fast, secure and decentralized transactions. The Blockchain technology is used in virtual coins (bitcoin) conditions, with a high innovation potential, applicable in various areas, with the advantage of storing databases, resulting in an unprecedented level of transparency in the private or public area. Interestingly, under the bitcoin conditions, the black chain system uses a decentralized peerto-peer payment system. Practically, the bitcoin can be considered as the most appropriate triple game accounting system. All of these considerations are developing in the big data era, which is defined as a large, diverse, high-volume information base requiring new forms of processing. Big data is important for businesses because based on these, strategic and marketing decisions can be made to optimize the activity in the market conditions and consumer preferences. European Union directives provide for measures to ensure the development of all states and, in this context, the community. At the same time, some measures provide for a more accelerated development for states with a low accession. For this, funds have been made from which important amounts are allocated to these states. The complex development of the European Union aims, in fact, to improve the quality of life (standard of living) in all Member States. At the European community level there are databases usable in economic analyzes. Also, Eurostat is the institution with the most complex databases. Recently, the Conference of the Directors of the Institute of Statistics in the European States analyzed the perspective of calculating the indicators in the context of the big data to be implemented. The article focuses on the concrete study of the use of large data in the calculation of the indicators that underlie the comparability between the EU Member States.
\end{abstract}

Keywords: Blockchain; bitcoin; finance; peer-to-peer; big data; strategy, community funds.

\section{Introduction}

In this article, the authors attempted to highlight the perspective of the evolution of the global settlement, payment system, and so on. Emphasis is placed on describing the Blockchain 
objectives and on setting the elements which give supremacy of the system in the future.

In this way, there is talk of speed, low costs, the elimination of errors and central attack and crash points, and the elimination of the risks involved. The platform allows a timeharmonization of the digital recordings, resulting thus that the Blockchain has all the details, ranging from fairness to transparency as regards the respective activity.

In the emerging world of the Blockchain, the issue of trust is an important one and it is widely described in this study. It is also noted that at present there is already a structure that controls the Blockchain and this, under the Big Data conditions, is the system that will surely ensure a great evolution.

Also, the institutions that occupy dominant positions may turn around the Blockchain if they find a leadership that focuses Big Data on structures and elements that respond to requirements, that is, to be able to solve problems based on the algorithms concerned.

The study shows that the Blockchain solves problems of general and particular interest, it is the fastest way to ensure for the created platforms a very special operativity.

In the context of the growth of the Peer-to-Peer global platform, trust and rationality is ensured for using the Bitcoin-based Blockchain system.

The fact that Blockchain, through the huge database, manages to provide trillions of transactions increases the confidence of those who will use this system. This Blockchain system has network integrity, distributive power, incentive value, preservation of the rights of the data owners and privacy, which implies that the other users' access is limited when accessing these transactions or operations.

Finance is monopolistic, but valuation and trading, especially through the banking system, are all in the field of the Bitcoin-based Blockchain. The study shows that the future financial services sector will undergo some changes that will lead to the authentication of the identity and value, rapid value movement, stocking values, borrowing and trading of values, but also changes in financing and investment fields that gain an ample field of analysis.

This study is, in a way, a great novelty element in the Big Data era, which implies, however, the transformation of the statistical procedures and activity into a readily accessible, understandable way.

\section{Literature review}

Anderson and Semmelroth made a study on the big data role on international economic analyzes. Anghelache, Anghel and Bodo (2017) analyzed how information helps to improve decision-making models, while the dynamics of information and the emergence of more and more additional aspects make the modeling process a continuous process, constantly expanding and adapting. Anghelache et al (2016) present the foreign trade level of Romania. Anghelache, Stanciu and Prodan (2014) conducted a comprehensive study on the process of producing IT goods and services, insisting on the issues of security for their use. Barber, Boyen, Shi and Uzun (2012) have conducted a study on the effects of Bitcoin and have tried to identify how Bitcoin could become a good candidate for a stable long-term currency. Bohme, Christin, Edelman and Moore (2015) presented the Bitcoin, studying its uses, risks and regulatory issues, starting from the consideration that Bitcoin will be the future. Bris, Koskinen and Nilsson (2008) study the corporate valuation. Cárdenas, Manadhata and Rajan (2013) highlighted the need to identify new ways to mitigate the impact of sophisticated attackers on the use of large data to change security tools for network monitoring and event management. Catalini and Gans (2016) used economic theory to analyze how the block 
technology will shape the innovation in digital platforms, identifying two key technologyrelated costs, namely cost of verification and network cost. Esposito, De Santis, Tortora, Chang and Choo (2018) examined the potential to use Blockchain technology to protect cloud-hosted health data. Eyal, Gencer, Sirer and van Renesse (2016) presented a new Blockchain protocol called Bitcoin-NG (Next Generation) and robust to extremes. Kennan (2017) studies the effect of migration for E.U. Koulakiotis et al (2012) analyse the GDP in EU and the influence of price evolution. Grinberg (2011) examined some relevant legal issues related to the use of the Bitcoin digital coin, as long as it is not supported by any government or other legal entity, relying on peer-to-peer networks and cryptography, and maintains integrity. Lafuente (2015) addressed a number of issues regarding the legitimate use of large data and customer confidentiality, highlighting that before starting any large data projects, the entities must have the appropriate mechanisms to protect these data from the very beginning. Lewenberg, Bachrach, Sompolinsky, Zohar and Rosenschein (2015) analyzed the effects of using Bitcoin, which is a decentralized innovative crypto currency which security is based on a procedure that requires network participants to repeatedly calculate entries in a large search space. Reid and Harrigan (2012) showed that in Bitcoin, a peer-to-peer electronic system of electronic values, anonymity is a delicate and complicated issue and conducted a study based on a non-trivial topological structure, information and external techniques. Yli-Huumo et al (2016) studied the emergence and impact of Blockchain technology, as well as issues related to improving Blockchain's limitations from confidentiality and security. Tschorsch and Scheuermann (2016) examined the basic structures and prospects of the Bitcoin protocol and its applications.

\section{Research methodology, data, results and discussions}

As well known, the Blockchain technology is used in the context of virtual coins, such as bitcoin, but has much greater potential to innovate, applicable in many areas.

The advantage is that it can hold a public data shared areas, especially now, in the era of Big Data, where there is a large amount of data on which basis analyses can be made, a lot of issues that have significance in the financial and economic area can interpret be interpreted.

The big banks and some governments implement Blockchains as distributed registers to revolutionize the way in which the information is stored and the way in which the financial, economic, etc. transactions take place.

The Blockchains' objectives consist of rapidity, lower costs, security, fewer errors and the removal of the central attack and crash points, of penetration into the system.

These models do not necessarily involve crypto-money for making payments. Bitcoin software or other digital currency are not stored in any file, they are represented by transactions recorded in a block, in fact, uses the resources of a large network Bitcoin "Peer to peer" to inspect and approve each transaction with Bitcoin. In computer language, Peer to Peer is a direct connection between two computers in the same network that can share information without the need for a third computer to have the server function.

The Blockchain uses bitcoin. Absolutely, all the distribution blocks work online, being made available around the world. There is no central database to be broken. That's why the Blockchain is public, seen by anyone at any time, because it's in the network and can intervene. 
The platform looks for real-time digital recording of the digital records on all the financial transactions that are being made. It is clear from this that the Blockchain has all the details, having the transparency necessary for the person who is interested to enter and find the information that interests him or her to do or not the transaction.

We can note that the Blockchain is, in our day, what the Internet represented at the time of its appearance a few decades ago.

The investments in "start-up" companies based on Blockchain are starting to thrive, develop as much as investing in site-based companies in the 1990s. Those who invest venture capital show interest as, by the way of acting the Blockchain they have the assurance that the transaction, the partnership created, the achievement of the investments and everything else is done clearly as they wish. The "start-up" is a newly established firm with a very rapid development which meets a market demand by creating or offering an innovative product, process or service.

Regulatory bodies are studying and enforcing Blockchain legislation in this area to avoid the emergence of the trade-threatening risks as well as the changes of the trade transfers.

Blockchains have gained a lot in the digital age as they provide concretely the main principles that every investor or any company that enters into the transaction needs. Thus, honesty is not just a matter of ethics, it becomes an economic problem, in the fact that the transactions must be made by partners with capacity, potential partners, good faith partners who work efficiently, that is to say quickly, to complete that work.

Consideration often means a fair exchange of benefits or advantages and disadvantages between the parties, each partner studying carefully how the transaction will end and the results it gets.

The Blockchain also ensures transparency, meaning that any transaction, any operation is done in the light of the day, without any alterations in hidden or difficult to identify elements.

In the emerging world of the Blockchain, trust derives from the network, even from the network's objectives, in the sense that, in order to avoid confusion, confidence refers to the purchase and sale of goods and services and the integrity and protection of information. Sure, this means protecting information against third parties.

If we remember, in the Internet era there was the question of energy and the spirit of convergence. The Internet assures rapidity in transmitting opinions, solving the problems that are trailing and that may harm the functioning of the Blockchain. Nowadays, we can also talk about the Internet as a possibility of transmitting false data but, anyway, the element of progress remains.

The Blockchain is similar to a superior stage, providing sightseeing transactions that ensure the rapid realization in the interest of those who succeed in making these transactions.

Presently, there are institutions that control and hold the means of production and social interaction, infrastructure, and everything else, and the Blockchain is the system that can become quite efficient and secure in doing this.

We can also talk about the fact that the enormously voluminous database in the Big Date era provides simplification by using the Blockchain that selects, captures valuable data collections which it provides to those interested. 
The Government institutions use the Internet to improve their operations and services, but now they are using Blockchain technology as well, which ensures the speed, transparency and use of the bulky database by the selection made for the desired purpose.

With the advent of Blockchain technology, a whole new world of openness has opened up to reverse all these trends in the sense that there is a peer-to-peer platform that allows for many large volume transactions, all being done with honesty, transparency and, to say so, the right interest, correct interpretation, for those who apply to them.

Nowadays, an organization is required to be endowed with the right to provide complete data, clear data to those wishing to enter the system, and to identify multiplesourced trading outlooks.

The experts often talk about a number of platforms like AIRBNB, UBER LYFT, TASKRABBIT and others, as platforms for the economy of protecting the interests of those who apply to them.

At present, things are happening rapidly, and it is clear that the financial services industry is helped, in the conditions of the global economy, to go to this Blockchain system. Undoubtedly, the most centralized sector in the world and the last area in which this transformation has been felt is considered a real revolution in domestic and international trading. Being full of contradictions and uneven advances, this system is only used by a limited number of people only. It is used by a small number of people, but it will surely gain great expansion in the future.

The institutions that occupy dominant positions may turn around the Blockchain technology if they find a leadership that will be able to concentrate BIG DATA - the big data base that will ensure trading as desired.

The property rights are so linked to the capitalist market system that the first version is found in the Declaration of Independence and Inalienable Property Law. It is clear that most owners in the world can see their home or small property confiscated arbitrarily, rightly or unfairly resolved. The Blockchain program, providing a huge amount of data, quickly returns to the concrete situation, without delays, as is currently the case for commercial litigation, lawsuits and many other impediments.

The central idea of the Blockchain is that the property rights can be traded regardless of whether they are financial, physical assets or ideas. The objective is not only to register the ownership, but to consign to the database, documents, attributes of the property, and its immutability.

The largest flow of funds to the developing countries is represented neither by external aid nor by direct foreign investment. In reality, it is the flow of money transferred, repatriated to the poor states by the people who create them.

The Blockchain solves problems of general interest in the sense that, when appealing to it, it has a database to interpret the right of the person to whom it belongs. The Blockchain can improve the distribution of the external aid by removing the intermediaries who take the aid before arriving at the destination, and who, by doing so, develop the vast array of those who in this process of helping the poor create great properties for themselves.

Also, as a permanent register of the funds circulation, the Blockchain determines the institutions to become more accountable for their actions. We remember that, during the first generation of the Internet, many intellectual property creators were not adequately rewarded, on the contrary, they were relied on to benefit from this right. Since then, from the Internet phase to the Blockchain, little has changed. 
Until now, we find that a series of corporations' reconfigurations have emerged as a driver of the capitalism. In the context of the development of a Peer-to-Peer global platform for identity, trust, reputation in transactions, we finally find that it is necessary to redesign the profound structures of the companies for innovating, creating distributed value and even of properties belonging to many and not only to a few.

We can also talk about the cultivation of the Blockchain entrepreneurship. The entrepreneurship is essential for a prosperous economy and society. The Internet has to release the entrepreneurs by offering them the tools and capabilities of the large companies, leaving aside obligations such as the inherited culture, oscillating processes, and all other elements that hinder progress.

The problem is complex, but the Blockchains can contribute to over-feeding the entrepreneurship with a solid information base and data, and therefore to ensure proper transactions as entrepreneurs.

We can also address the issue that the Blockchain, through the huge database and tracking the transaction spontaneously, very quickly, ensures the installation of government authorities by the people for the people. There is enough discussion about whether a governmental institution is representing the interest of the entire population, that is, of the whole people. The Blockchain technology requires a culture and training level for the society members who can access and quickly see how the transactions evolve, how these issues are going.

Under the current circumstances, we can also discuss the prospect of initializing the future, taking into account the seven design principles of the Blockchain economy. The seven design principles are considered to be necessary in the context of discussing a Blockchainbased system.

The first is the network integrity. This principle is based on trust that expresses the possibility that everyone who wants to enter this system can use it for decision-making and information. As a rule, people were not able to trade or do business directly on the Internet simply because money does not resemble the other information assets and intellectual property of the sites. That is why the Blockchain marks a remarkable progress in this direction, unifying the prospects for progress, imposing a certain strategy seen and recognized by all those who are turning to this system in full development process.

A second principle is the distributive power, in the sense that the system can distribute and can use or be used by all those interested.

In other terms, the third principle would be the value as an incentive, in the sense that balancing and correlating the values of the transactions, of the traded items, it ensures the possibility of performing efficient operations.

According to the fourth principle, the Blockchain system ensures the security of the system in the sense that the access is available only to those who are directly interested in it and entered into the transaction.

The fifth principle is the confidentiality, which implies that the access of the others is limited in the course of this transaction or operations.

The Blockchain also assures, through the sixth principle, the conservation rights, that is, the right of ownership, which is imprescriptible and cannot be used because this Blockchain system stops you from going further.

The last principle of the Blockchain's economy is the inclusion, which assumes that the Blockchain encompasses the entire informational base. 
The global financial system moves millions of dollars a day and serves billions of people, sustaining a global economy worth over \$100 trillion. This is the world's most powerful industry, the foundation of the global capitalism, and those who hold the funds, who have these huge sums, are considered the masters of the world.

The population and resources are the basic elements that provide insight into the evolution of mankind, but also involve a more careful analysis.

Currently, the world's population is over 7 billion people. In 2050 there will be around 10 billion people. The resources, however, are exhausted. Of course, there are prospecting, there are analyzes that point obviously to the prospect of other resources, but thinking about how these resources are consumed has to be very profound.

Everything is going through the global financial system and this one should play an important role, because so far, but from now on as well, the world's states will be divided between rich and developing or even poor countries.

This Blockchain system provides enough information to show how the global transactions take place, the transactions between states, and so on. The monetary policy makers and financial regulators are often out of the planning capability of large financial operations, and it is therefore difficult to understand the evolution of the mankind as a whole.

The 2008 global financial crisis has been an eloquent example. An excessive influence, namely the lack of transparency and a sense of self-esteem put into motion, stimulated and distorted the world situation. Of course, the monetary mass in circulation, over its capacity to cover it with goods, was the element that determined the crisis that I mentioned.

The installation of new technologies over the existing infrastructure is an anticipated fact and therefore, in the next period, the importance of the Blockchain will be more obvious, which, based on the principles we have mentioned, will represent the possibility to settle many international transactions.

Finance is monopolistic. In their assessment, during the financial crisis, it was established that the banks were doing everything they could to increase the transaction costs in any way. Even at the retail level, the payments for basic goods and services have grown steadily and created the impossibility of the large mass of the population to be able to solve their daily problems. The public finance days are being counted now, when the Blockchain promises to make the next decade one of the great upheavals and transformations, as well as the tremendous opportunities for those who will succeed in taking advantage of them.

The global financial services industry is accompanied by a number of problems, it is built on an old technology that has been in operation for several decades and, therefore, in the digital world, which is advancing very rapidly, there will be the issue of major transformations in this field to consider.

There are six key reasons, explaining why the Blockchain technology will cause profound changes in the financial sector, breaking the monopoly of finances and providing both the individuals and the institutions with a real choice of how they manage value, because everything is needed be based on value, on evaluation, and then on value expression.

The financial services industry is a lot of inherited technological systems that is about to get destabilized if the Blockchain we are talking about does not consolidate.

In the future, the financial services sector will suffer a number of changes. First of all, the authentication of identity and value will certainly arise. Currently, there are strong intermediaries in transactions that usually set the financial transactions. These intermediaries are those who know all the details and all the sensitivities of the banking 
system. The Blockchain reduces and sometimes completely removes the confidence in certain transactions. Therefore, the role of the intermediaries in international transactions is somewhat diminishing.

The movement of value represents another change in the financial system. The value will move every day, the money being that to facilitate the compensation, settlements and all the elements that are needed in the case of the international financial and economic transactions.

Then we can talk about the storing value, meaning that the financial institutions are valuable deposits for individuals, institutions and national authorities. In the Blockchain, there is the possibility of bringing all the values to light, we are here referring to the worldwide monetary mass that the world's economy has.

Another feature is the lending value, meaning that from mortgages to treasury bills, financial institutions facilitate the credit issuance such as credit card claims, mortgage loans, corporate bonds, municipal bonds, government bonds and asset-backed securities.

The credit sphere has led to the emergence of new auxiliaries who carry out credit checks and calculates credit scores and credit ratings.

Another feature, a change that will occur in financial services, is the transaction of value. Every day, markets globally facilitate the exchange of financial assets at the level of trillions of dollars. But not every day, but every minute, because of time zones, transactions take place in every part of the globe. The transaction refers to the purchase and sale of financial assets and financial instruments for investment purposes, financial speculation, risk hedging, and international monetary and financial arbitration. The Blockchain reduces the settlement time for all the transactions, from a few days and weeks to a few minutes or seconds.

Here we infer another change in the financial system, namely in the area of the finance and investment. Investments in an asset made by an entity in another new entity are made on the basis of opportunity, studies, protocols and other significant documents. In the context of the Blockchain well set up, these investment transactions, which are the key to the economic growth and international developments, it becomes easier to make such transactions.

Another modification, another definite change that will be imposed by the Blockchain, concerns the value-assurance and risk management. The risk management, of course, includes insurance that has the objective of protecting entities against losses that may occur.

As a last estimate, the national value of all current OTC derivatives is over 600 million dollars. The Blockchain supports and provides decentralized insurance models, making the use of the derivative instruments more transparent for the risk management.

Last but not least, we can talk about value accounting. In this sense, the Blockchain will make these cost accounting highlights instantaneous, i.e. to express the ability of the regulators and other stakeholders to thoroughly verify the financial actions carried out within a corporation.

The Exchange trades become particularly sensitive when they are made in the Blockchain system.

The financial benefit of this Blockchain is also revealed by the fact that in 2015, 9 of the world's largest banks (Barkley's, JP Morgan, Credit Suisse, Goldman Sachs, State Street, UBS, Royal Bank Scotland and Bank of Commonwealth) entered in a standard collaboration 
plan through Blockchain technologies. These have facilitated the interbank activity as well as the market activity.

We can also talk about the initial Blockchain public offer. Because of the new multiplication platforms, the small companies can gain access to capital, but they also have a chance to access it. The Blockchain ensures extensive participation, and it is expected that Blockchain's public bids launched on platforms will change the way in which they are made. Also, the derivative market is located in the the Blockchain with an impressive volume of data, Big Data, which provides the possibility of forecasting, long-term forecasts in the most private areas of activity, as well as at the global plan.

Consequently, the Blockchain, as it is defined, will be for the world of finance comparable to what happened 25 years ago when the Internet captured the world of information.

\section{The economic evolution of the EU Member States in the big data era}

The main objective of the European Union is the individual development of all the member countries and, in this way, the development of the European Union. The European Union comprises two groups of states. Some who are members of the euro group and others who are not members of this group. Of course, for the states that are members of the Eurozone, things become much simpler, in the sense that the whole economic evolution, reflected in the accounting standards, shows in entries and exits that finally give the possibility of calculating the indicators gross domestic product and gross domestic product per head. resident in a continuous manner. States that are not members of the euro group, including Romania, have a different situation, because on the one hand internally gross domestic product, gross domestic product per inhabitant and other macroeconomic indicators of results are calculated, based on accounting records, in The System of National Accounts, expressed in own currency. When making Eurostat the data series that ensure intra-European comparability, the exchange rate is used to bring the mentioned indicators from the value registered in the national currency into the European currency. Therefore, these states normally but also paradoxically have two evolutionary curves: the first is the one given by the increase expressed in prices and value in the national currency, and others that depend on the expression in the euro. Therefore, we can talk about one evolution or another of each Member State.

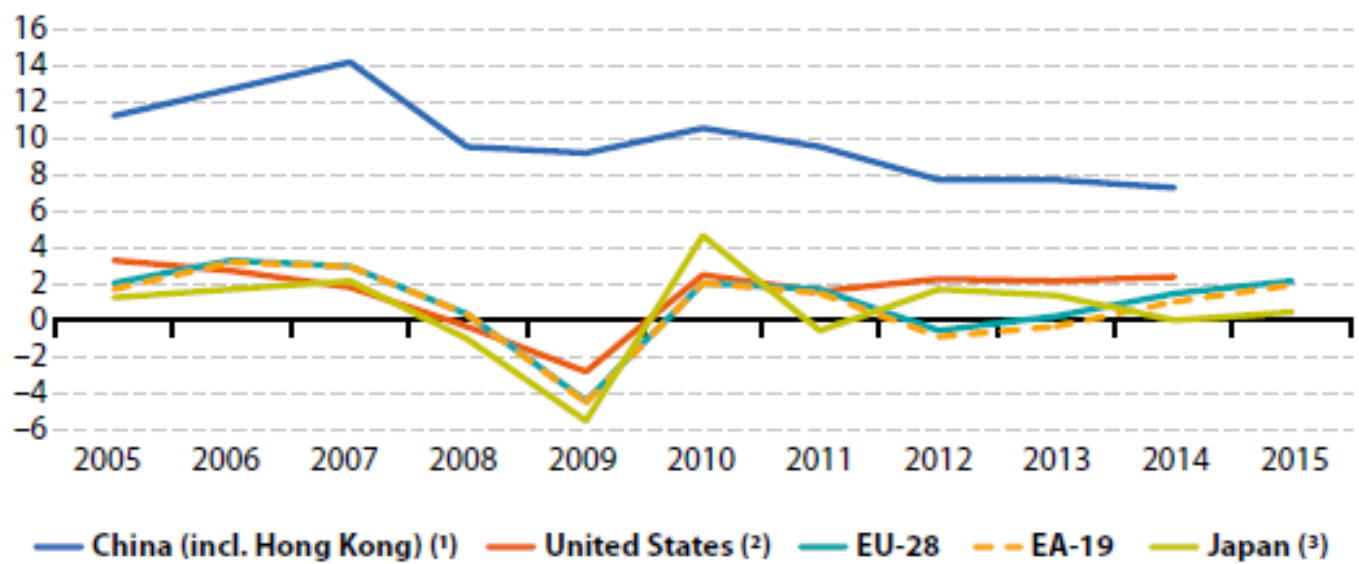

Figure 1. Real GDP growth, 2005-2015 (\% change compared with the previous year) Source: Eurostat - Key figures on Europe 2016, pag. 89 
Of course, the growth of the gross domestic product in the period 2005-2015 is expressed and mirrors exactly what we mentioned in figure number 1 .

From this figure it is very clear that a number of states have had a certain evolution which, if we compare it with other states, give some deviations. Also, in Figure 1, in which we have presented the evolution of the Member States of the European Union, compared to other states such as China, the United States or Japan, we observe certain evolutionary levels, and these lead to a pragmatic interpretation on the way the European Union evolves. as a whole, as well as the Member States of the European Union. The analysis of the economic growth of the member countries of the European Union must be treated differently. This is because there is the possibility of household contributions in some countries, and in others it may be insignificant. The member states of the European Union, of course, have different growth rates, but it is important for each of them to ensure a significant increase, which will contribute to the growth of the gross domestic product per inhabitant as well as the standard of living in general.

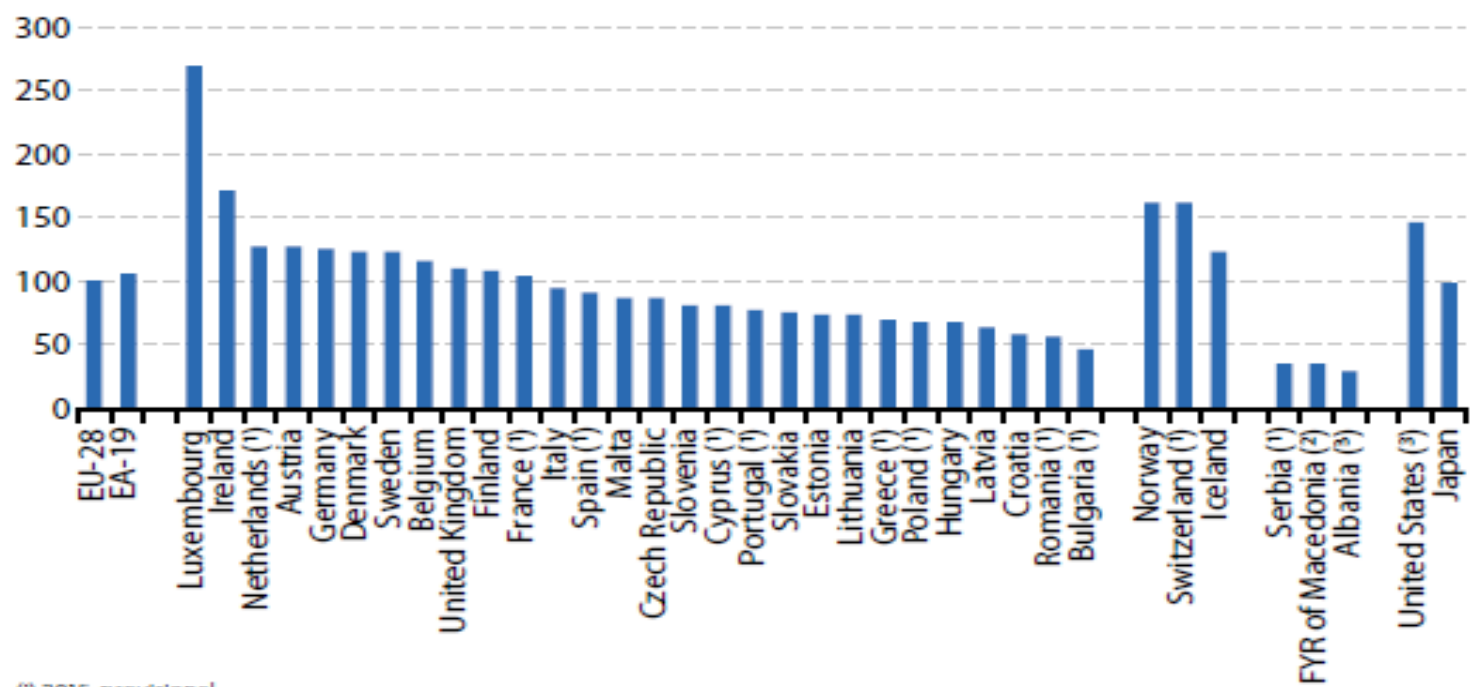

Figure 2. Gross domestic product / capita in current prices, 2015 (EU-28 = 100; based on PPS per inhabitant)

Sursa: Eurostat - Key figures on Europe 2016, pag. 90

In the figure no. 2 we have represented this growth trend, with the possibility, by simply interpreting the data contained in the graph, to identify the level of growth that each of the member countries had, as well as of the European Union as a whole. It is noted that the member countries, as well as those of the euro group, have had reasonable increases, while some countries such as Luxembourg, Ireland, the Netherlands, Austria, Germany, Denmark, Sweden, Belgium and even Finland have increased slightly above $100 \%$, this pointing out that always, wherever it is invested, where there is a harmonized economy, I mean the branches of the national economy, the growth is also tailor-made. Some of the countries, including Romania and Bulgaria, have a very low gross domestic product per inhabitant. To these are added Lithuania or Croatia. It is important to note that all member countries have a possibility of sustained growth in subsequent periods. 


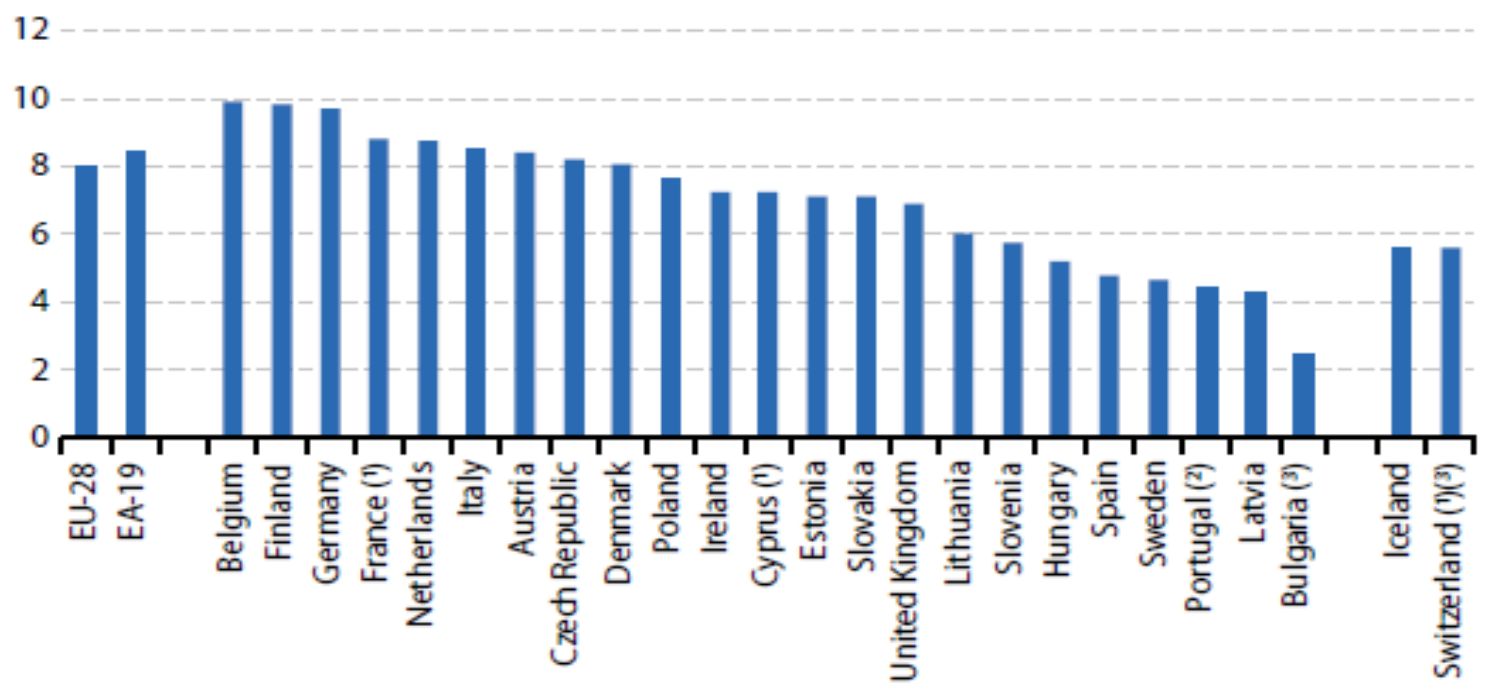

Figure 3. Gross investment rate of households, 2015

Sursa: Eurostat - Key figures on Europe 2016, pag. 91

Next, based on the data in figure 3, namely, the gross investment rate of households in 2015, let us find out what is the contribution of the investments made at this level. As a result, a number of countries, including Belgium, Finland, Germany, France, the Netherlands, Italy, Austria, the Czech Republic, Denmark and Poland, have increased by over $8 \%$ or around $8 \%$. Other countries, such as Bulgaria, Lithuania, Portugal, Sweden, have risen from 2.2 to $4.5 \%$, which is not bad, given that the contribution of this sector of activity has been reasonable enough. From the point of view of our country, we find that in this chapter of the analysis, Romania is located somewhere in the middle area, with a share of $56.9 \%$ of the total growth in other sectors.

\section{Government financing}

The government funding is an essential element in the analysis of the economic growth of the member countries of the European Union. Of course, government funding refers to both possibilities, namely financing for the construction and finalization of the state budget, the consolidated budget, as well as financing for investments. From this point of view we can see that in the member countries the indicator that is calculated represents the deficit of the annual budget. According to the directives of the European Union, this annual budget cannot have a deficit greater than $3 \%$. Of course, for countries with a balanced economy, a welldeveloped structure and where the financial and non-financial market sector are contributing, meeting this parameter is not a particular problem. However, in other countries, including Romania, the question arises to make some budget restrictions in order not to exceed this percentage of 3\%. However, most countries in the European Union have reasonable growth weights, but there is a danger that some countries may have much higher growth. According to the European directive, the countries that exceed the percentage of deficit mentioned above will have positive results in terms of economic growth. The government debt in 2014 and 2015 was different from one country to another. Within the European Union, as a whole, the level of accumulated debt was $85 \%$. We can appreciate the fact that it is not a problem that debt accumulates, internal or external, in the context in which the economy operates at the parameters that it must acquire in the respective periods. 
However, there are some discrepancies when there is no such possibility and then the debt increase is quite delicate. An indicator showing the level of internal and external public debt growth is the indicator of the weight of debt in the annual gross domestic product of a country. This is a parameter that shows how countries are indebted and the difficulties they face in a possible crisis or situation that is unfavorable at European, or worldwide, level.

\section{Exchange rate and interest rate}

Regarding exchange rates and interest rates in the European Union, we find that countries, depending on their membership or non-membership of the euro group, also get different results. Thus, most of the member countries of the European Union that are also members of the euro group do not have difficulties in obtaining reasonable results.

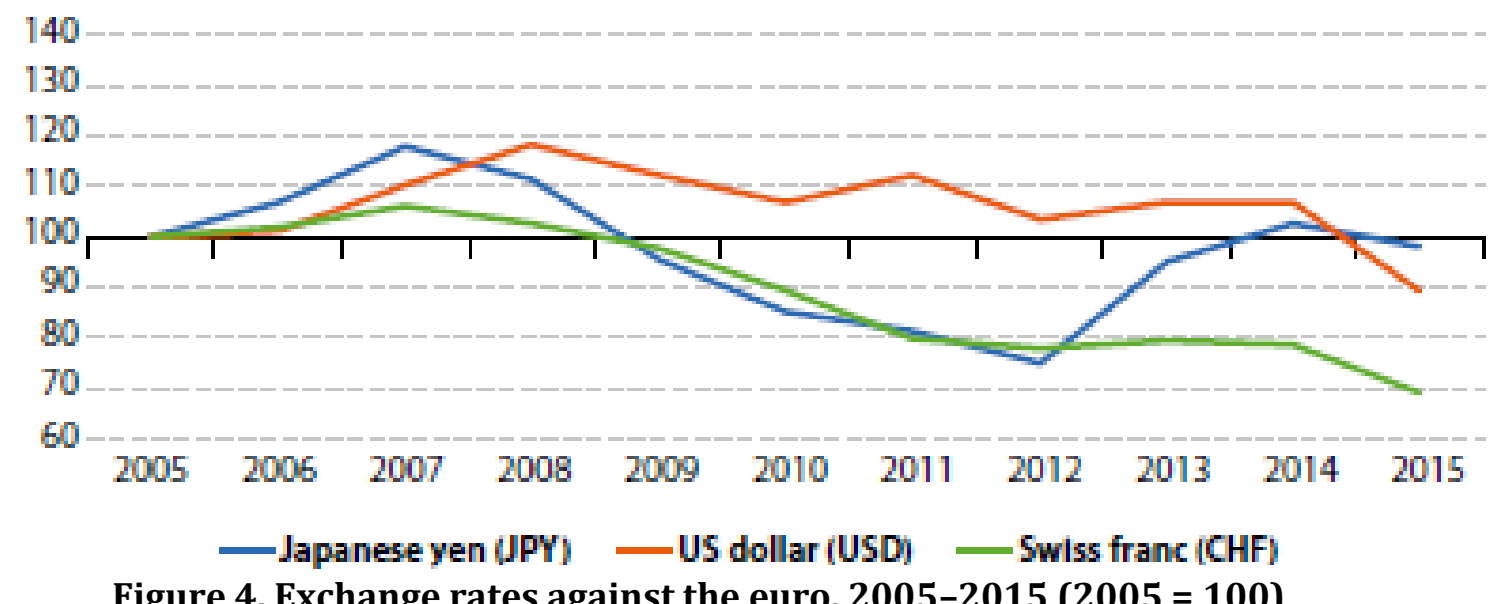

Figure 4. Exchange rates against the euro, 2005-2015 $(2005=100)$

Sursa: Eurostat - Key figures on Europe 2016, pag. 95

In the figure no. 4 , we also presented the exchange rate situation against the euro in the period 2005-2015, following the evolution on three levels. One is the Japanese yen, the second is the US dollar or the Swiss franc. We find that this exchange rate against nonEuropean currencies is different but it has always had a downward trend. It was found that during the period 2012-2015, the Japanese yen had a decreasing trend in relation to the euro. We initiated this analysis because, in essence, the member states of the European Union carry out intra and extra-Community trade. When carrying out non-EU activity, attention must be paid to how this balance is achieved, by exchanging currencies. We are not talking here about financial-currency arbitrage, but only about international trade operations carried out in the non-EU system.

\section{Effect of inflation on comparable price levels}

Another important element is the fact that the inflation rate plays an important role in the Member States of the European Union. In this respect, the data used are comparable. We presented the situation of each country according to the inflation rate, based on the transformation of each of the indicators into real terms by deflation. 


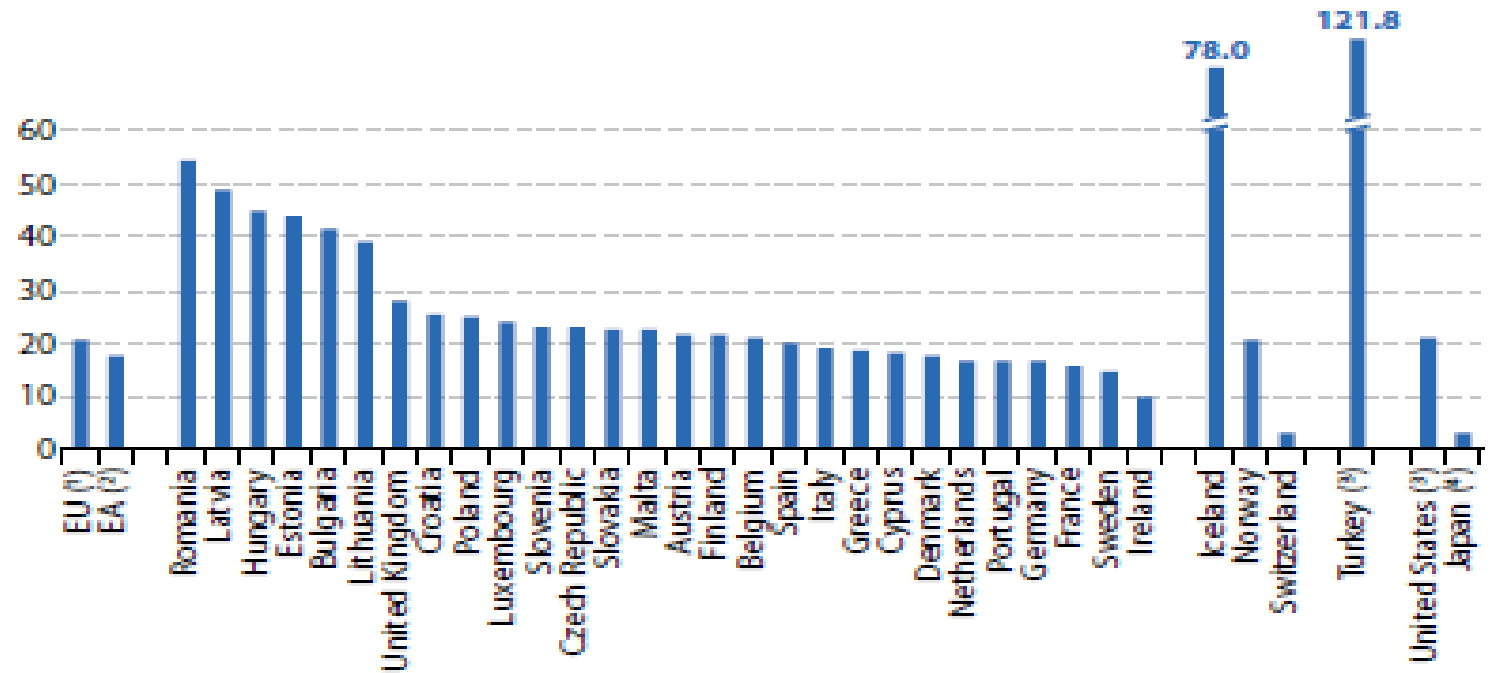

Figure 5. HICP all-items, inflation rate, 2005-2015 (\%)

(1) The data refer to the official EU aggregate, its country coverage changes in line with the addition of new EU Member States and integrates them using a chain-linked index formula;(2) The data refer to the official euro area aggregate, its country coverage changes in line with the addition of new EA

Member States and integrates them using a chainlinked index formula; (3) Definition differs; (4)

National CPI: not strictly comparable with the HICP 2014 data.

Source: Eurostat - Key figures on Europe 2016, pag. 97

The deflated data give the situation presented in this figure no 5 , in which we find that in Romania the influence of inflation is higher than in other countries.

\section{Balance of payments in the European Union}

Next we referred to the situation regarding the balance of external payments within the European Union. The balance of payments within the European Union is one that shows what debts are created for a country if imports exceed exports. It is clear that in this situation in a number of states there is a destructive effect, in others it is more reasonable, more balanced.

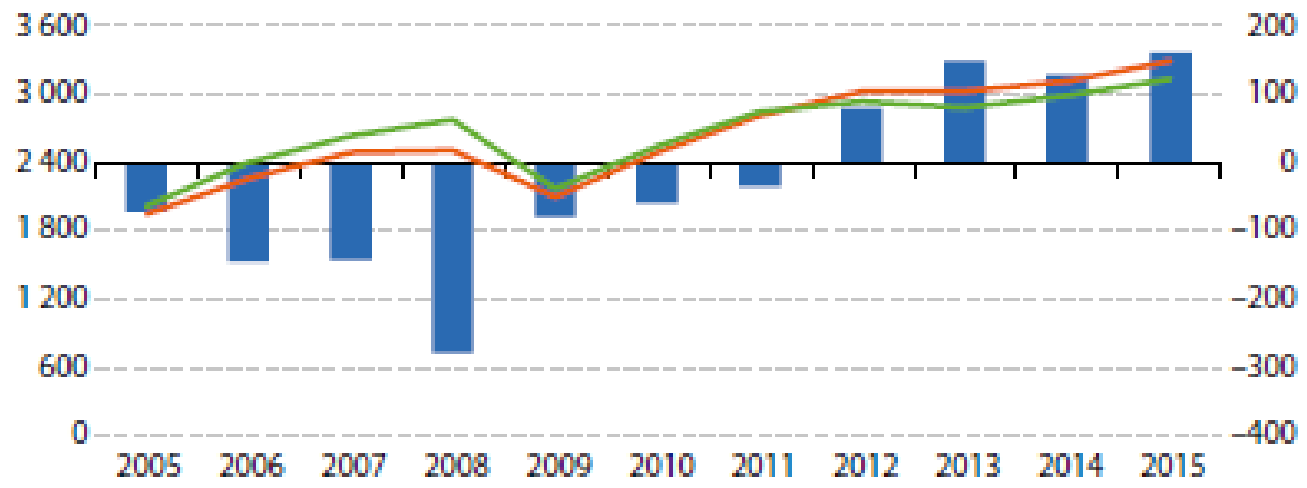

Balance (right-hand scale) _Credit (left-hand scale) - Debit (left-hand scale)

Figure 6. Current account transactions, EU-28, 2005-2015 (billion EUR)

Sursa: Eurostat - Key figures on Europe 2016, pag. 99 
We do not insist too much on this aspect because it turns out that by the way we presented the figures we mentioned in the economic evolution of the European Union member countries, we can also come off how these countries have achieved a balance of external payments balance, a balance. which has also been transferred in the growth of the gross domestic product and not in the last instance and the possibility to make greater investments or have a role to increase the incomes of the population to ensure a standard of living, as the European Union also states through its directive, balanced growth in the respective country.

\section{Conclusions}

From the study conducted and presented in this article a series of conclusions can be drawn. First of all, in the Big Data era, there is an abundance of data and information that is stored and can be processed and used to achieve rapid decision-making in this way.

In the financial and monetary system, more and more emphasis is being placed on the use of the virtual currency to replace the material transfer in the realization of the monetary mass. In the so far systems we have seen also the possibility of using the transactions paper, in the sense that they moved only the documents without the need of the flow of money that actually moves, as it entered only in the settlements on transactions, on banks, on operations and so on.

The Blockchain is the system that ensures the concentration and then the processing, analysis and interpretation of data from the Big Data bases. The huge number, in the order of thousands of trillions, of information and data, no longer makes it possible to use office computers or the individual work of civil servants. From this point of view, the Bitchin-based Blockchain system will be the one that will ensure the transaction speed and all the rest.

The transactions in this era, the third millennium, have an extraordinary range and field of operation. There are enormous distances, many are not even known to each other, but they can only trade through Bitcoin's Blockchain system.

Although this issue of the Blockchain in the Big Data appears a bit fanciful, it will be the one that replaces all the operating systems so far and will thus ensure the leap that mind and humanity accomplishes. Already there are a number of algorithms specific to some areas or general ones, which make it easy and secure the evolution of this system, of this creation, let's say it so.

The authors' study reveals a series of conclusions regarding the content and significance of the economic analysis in the context of the big data. Thus, in the field of financial services and insurance, this system is irrefutable. Financial institutions could use intelligent devices to mark their rights over physical assets, which can be traced and detected. Digital models allow fast storage and transfer of data and value for all users, regardless of their size. In this way, the mood arranger has also assessed the risk perspective and, in other words, in the financial-banking-monetary field it ensures the perfect risk management.

It is clear that in banking, the pre-analysis of credit systems is astonishingly simple by using this blockchain. This economic development of each state is easy to achieve on the basis of data provided by Eurostat, which are increasingly based on the big data.

In the field of insurance, it is ensured that the payments are adjusted according to the place where the object and its environment are located, so that there can be no delays, because the calculated algorithms will immediately solve these problems. 
As the outcomes can easily result, it is obvious that physical assets can quickly become digital assets. All documentation related to a particular asset can be digitized and brought to Blockchain. In this sense, the same can be done with the patents, property, guarantees, certification inspections, provenance, insurance, replacement data, approvals, etc., thus unimaginably increasing the possibility of having an operation, a complex transaction, done in seconds, if not sooner. The economic analysis and development perspectives of the European Union, based on the huge amount of data, suggest precisely the trend that studies should be carried out on the basis of large data bases.

\section{References}

Anderson, A., Semmelroth, D. (2015). Statistics for Big Data, John Wiley \& Sons, New Jersey. Anghelache, C., Anghel, M.G., Bodo, G. (2017). Theoretical aspects of the role of information in the process of decisions/risks modeling. Romanian Statistical Review Supplement, 6, 102-111.

Anghelache, C., Anghelache, G.V. and Anghel, M.G. (2016). Analysis of the Evolution of Romanian Foreign Commercial Exchange. Romanian Statistical Review, Supplement, Issue 3, pp. 82-90.

Anghelache, C., Stanciu, E., Prodan, L. (2014). Production and marketing of IT goods and services. International Symposium "Programs for Romania's Economic Recovery in the „Horizon 2020” Perspective”, December 2014, Bucharest, 120 - 126.

Barber, S., Boyen, X., Shi, E., Uzun, E. (2012). Bitter to better - How to make bitcoin a better currency, Proceedings 16th International Conference Financial Cryptography and Data Security (FC'12), 399-414.

Bris, A., Koskinen, Y. and Nilsson, M. (2008). The Euro and Corporate Valuations, Review of Financial Studies, 22(8), 3171-3209.

Bohme, R., Christin, N., Edelman, B., Moore, T. (2015). Bitcoin: Economics,technology, and governance. The Journal of Economic Perspectives, 29(2), 213-238.

Cárdenas, A., Manadhata, P., Rajan, S. (2013). Big Data Analytics for Security. IEEE Security \& Privacy, 11(6) , Nov.-Dec. 2013, $74-76$.

Catalini, C., Gans, J. (2016). Some Simple Economics of the Blockchain, NBER Working Paper No. 22952.

Esposito, C., De Santis, A., Tortora, G., Chang, H., Choo, K.K.R. (2018). Blockchain: A Panacea for Healthcare Cloud-Based Data Security and Privacy?. Cloud Computing IEEE, 5 (1), 31-37.

Eyal, I., Gencer, E., Sirer, G., van Renesse, R. (2016). Bitcoin-NG: A Scalable Blockchain Protocol, Proceedings of the 13th USENIX Symposium on Networked Systems Design and Implementation (NSDI '16), March 16-18, 2016 • Santa Clara, CA, USA, 45-59.

Grinberg, R. (2011). Bitcoin: an innovative alternative digital currency. Hastings Science and Technology Law Journal, 4, 159-208.

Kennan, J. (2017). Open borders in the european union and beyond: migration flows and labor market implications, National Bureau of Economic Research, Cambridge, Working Paper No 23048.

Koulakiotis, A., Lyroudi, K. and Papasyriopoulos, N. (2012). Inflation, GDP and Causality for European Countries. International Advances în Economic Research, 18(1), 53-62;

Lafuente, G. (2015). The big data security challenge. Network Security, 2015 (1), 12-14. 
Lewenberg, Y., Bachrach, Y., Sompolinsky, Y., Zohar, A., Rosenschein, J. (2015). Bitcoin mining pools: A cooperative game theoretic analysis, In Proceedings of the 2015 International Conference on Autonomous Agents and Multiagent Systems (2015), 919-927.

Reid, F., Harrigan, M. (2012). An Analysis of Anonymity in the Bitcoin System. Security and Privacy in Social Networks, 197-223.

Yli-Huumo, J., Ko, D., Choi, S., Park, S., Smolander, K. (2016). Where Is Current Research on Blockchain Technology? - A Systematic Review. PLoS ONE 11(10): e0163477. https://doi.org/10.1371/journal.pone.0163477.

Tschorsch, F., Scheuermann, B. (2016). Bitcoin and Beyond: A Technical Survey on Decentralized Digital Currencies, IEEE Communications Surveys \& Tutorials. 18 (3), 2084-2123, 2016. 